52

massa principale e bastava questa piccola quantita di sostanza liquida conduttrice disseminata nella massa solida per produrre ben visibili fenomeni di conducibilita. Ma io non crederei cho quantità così piccole come quelle che costituiscono le impurità inevitabili delle sostanze facili a purificare e che ho cercato di ottenere pnrissime possano essere sufficienti esse sole a mantenere una così forte conducibilita come quella degli esempii sopra riportati nelle tavole I, II e III ; in alcuni casi le impurità potranno contribuirvi per una minima parte e certamente esse tendono a mantenere una certa conducibilità; ma non credo che esse sole possano essere la causa di conlucibilita così forti.

Del resto, con l'idee che si hanno generalmente circa la costituzione dei solidi reali in ispecie in vicinanza del punto di solidificazione, non repugna affatto ammettere che alcuni di questi possano ritenere una certa conducibilità elettrolitica anche solidi $\theta$ ad una certa distanza dal punto di solidificazione: anzi la proposizione contraria troverebbe pochi che la credessero.

Firenze 1. Giugno 1885.

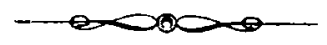

SULLA DIPENDENZA DELIA CONDUCIBILITÁ ELETTRICA DELLA DIETILAMMINA, DALLA TEMPERATURA; NOTA DEL PROF. ADOLFO BARTOLI.

( Latta alla R. Acc. dei Lincei nella seduta del 21 (Fiugno 1885) 1).

î noto già da lungo tempo che la conducibilità elettriea dei composti salini allo stato liquido) sia per fusione ignea, sia per soluzione) cresce colla temperatura, a differenza dei metalli nei quali il riscaldamento produce una diminuzione di conducibilità.

1) Noi Transunti della $R$. Accademia dei Lincei, fascicolo di giugno, pag. 572 dove questa stessa nota eे stata pubblicata, è incorso un errore di stampa assai rilovante, dicendovisi nel titolo dinretilammina in luogo di dietilammina. Ma nol corso di queila nota stessa ossendo stata seritta correttamente la formula $\mathrm{C}_{3} \mathrm{H}_{5}\{\mathrm{H}$ ed il punto di obulizione $+57^{\circ}$ del composto da mo studiato, è impossibile che il colto lettore non corregga da sè l'errore tipografico. Due aitri errori sono incorsi a pag. 573, dicendosi alla lines 13, etilammina in luogo di dietilammina, e all' ultima linea attila. mina invece di amilamina. 
Io ho constatato lo stesso fatto nelle diverse combinazioni del carbonio e dei metalloidi che ho fin qui studiato, e così pure nelle diverse soluzioni di questi composti, o nelle mescolanze che si trovano già formate, come gli olii essenziali, gli olii fissi, i grassi, le cere, i balsami, le resine ec. ec. ").

II. In questa nota descriverò il fenomeno singolare che presenta la dietilammina

$$
\left.\underset{\mathrm{H}}{\mathrm{C}_{2} \mathrm{H}_{3}} \mathrm{C}_{3}\right\} \mathrm{N}
$$

La dietilammina possiede una conducibilità assai notevole $\theta$ dell' ordine di grandezza di quella dell'alcole assoluto, ma la conducibilità della dietilammina decresce continuamente col crescere della temperatura. I limiti di temperatura fra cui ho sperimentato sono stati $-10^{\circ} \mathrm{e}+57^{\circ}$ ( temperatura di ebullizione). Il decrescimento di conducibilità entro questi limiti è stato assai rilevante.

Selnz' altro aggiungo qui i numeri ottenuti per la conducibilita relativa della dictilammina alle diverse temperature.

\section{Tavola I.}

Dietilammina preparata dal Kahlbaum di Berlino nel 1883-84. Bolliva $a+57^{\circ}(\mathrm{N}$. del registro delle esperienze 3803 ).

\begin{tabular}{cc|cc} 
Temperature & $\begin{array}{c}\text { Conducibilitì } \\
\text { relative }\end{array}$ & Temperature & $\begin{array}{c}\text { Conducibilità } \\
\text { relative }\end{array}$ \\
$-10^{\circ}$ & 10,790 & $+25^{\circ}$ & 7,18 \\
0 & 10 & +29 & 6,74 \\
+8 & 9,494 & +37 & 6,00 \\
+15 & 8,48 & +43 & 5,61 \\
+23 & 7,64 & +56 & 4,40
\end{tabular}

1) Compara la nota pubbliesta in questo stesso fascicolo del Nuovo Cimento, col titolo: La conducibilità elettrica delle combinaeioni del carbonio ed in ispecie quella delle ammidi, dei nitroderivati occ. occ. 
5.t

'Tayola 11.

Dietilammina preparata dalla nitrosodietilina. (N. del registro delle esperienze 3778 ).

\begin{tabular}{cc|cc} 
& Conducibiliti & & Conilucibilitil \\
Temperature & relntive & Temporature & rolative \\
$-1^{\circ}, 5$ & 10 & $+35^{\circ}$ & 6,00 \\
+7 & 9,35 & +43 & 5,37 \\
$+16^{\circ}, 5$ & 7,90 & +53 & 4,75 \\
+25 & 6,75 & +57 & 4,25
\end{tabular}

Risultati identici ai precedenti mi hanno dato altri due campioni di dietilammina preparati da un tempo piuttosto lungo.

III. Il fatto sopra enuociato ha cortamente qualche importanza ed è il punto di partenza di altre ricerche fisico-chimiche.

I campioni di dietilammina a cui si riferiscono le due tavole precedenti erano perfettamente puri e conserrati entro tubi chiusi alla lampada fino all' istante in cui si adoperarono; e ne furono studiate le conducibilita racchiudendoli entro un voltametro che si ebbe cura di chindere anche esso alla lampada appena introdottovi il liquido: inoltre il fenomeno si manifestara con pari intensità in due altri campioni di preparazione assai anteriore ma provenienti da preparatori diversi. Ma dato auche e non concesso che il fenomeno potesse da taluno attribuirsi ad impurità minime che per avventura fossero contenute nei liquidi esaminati, il fenomeno non cesserebbe per questo dall' essere inesplicabile (almeno per ora): dacchè nessuna dello tante soluzioni da mo studiate ha mai presentato un decrescimento di conducibilità coll' elevarsi della temperatura.

IV. Aggiungerd intanto, con la promessa di tornare fra breve sullo stesso argomento, che la propilainina $\mathrm{C}_{5} \mathrm{H}_{7} . \mathrm{NH}_{2}$ preparata dal Kahlbaum e bollente fra $+49^{\circ}$ e $+50^{\circ}$ serba fra $0^{\circ} \theta$ $+50^{\circ}$ la stessa conducibilità presso a poco invariata; c così pure l' allilamina $\mathrm{C}_{3} \mathrm{H}_{3} . \mathrm{NH}_{2}$ preparata dal Kahlbaum e bollente a $+58^{\circ}$ serba lo stesso contegno fra 0 e $+70^{\circ}$ (la temperatura di $+70^{\circ}$ fu ottenuta scaldandola entro tubo chiuso) e così pure si comporta fra $+20^{\circ} \mathrm{e}+80^{\circ}$ l' amilamina $\mathrm{C}_{3} \mathrm{H}_{14} \mathrm{NH}_{2}$ preparata da Kahlbaum e bollente $a+9 \breve{5}^{0}$.

Firenze 1. Giugno 1885.

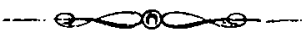

